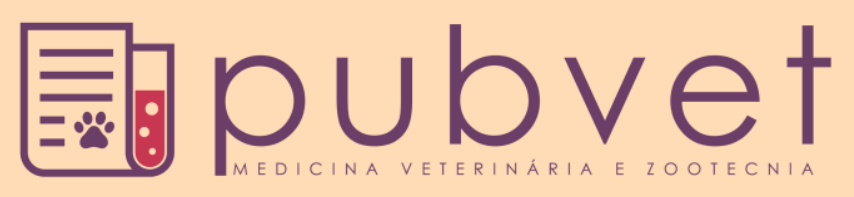

https://doi.org/10.31533/pubvet.v12n8a143.1-6

\title{
Ruptura do ligamento cruzado cranial de cães atendidos no Hovet da Universidade Anhembi Morumbi
}

\author{
Alane Leite Xalega ${ }^{1 *}$, Fernanda R. Almeida ${ }^{2} \bullet$, Silvia Ferrari $^{3 \oplus}$, Tatiana Helfenstein ${ }^{4}$ \\ ${ }^{I}$ Médica veterinária, São Paulo-SP, Brasil. \\ ${ }^{2}$ Estudante de medicina Veterinária da Universidade Anhembi Morumbi, UAM, Brasil. \\ ${ }^{3}$ Universidade Anhembi Morumbi, Setor de Reprodução animal São Paulo-SP, Brasil. \\ ${ }^{4}$ Universidade Anhembi Morumbi, Setor de Fisiologia Animal São Paulo-SP, Brasil. \\ *Autor para correspondência, E-mail: alanexalega@hotmail.com
}

RESUMO. O objetivo do presente trabalho foi identificar fatores considerados de risco, como raça, idade, sexo, peso corporal e castração em cães que sofreram ruptura do ligamento cruzado cranial (RLCCr), atendidos no Hovet Universidade Anhembi Morumbi. Foram pesquisadas as fichas dos cães com RLCCr atendidos no período de 2009 a 2015. Neste período foram atendidos 382 animais com RLCCr, dos quais 189 fêmeas e 193 machos. Não houve diferença estatisticamente significativa entre machos e fêmeas com RLCCr, entre idade da ruptura, entre os sexos e entre intervalo da castração e RLCCr, mas houve diferença estatisticamente significativa na idade à ruptura dos animais com peso acima de $20 \mathrm{~kg}$ em relação aos demais. Concluiu-se que há influência do peso do animal na ocorrência da RLCCr, pois animais com mais de $20 \mathrm{~kg}$ apresentaram a RLCCr com uma idade inferior à dos animais de porte menor. As raças mais acometidas pela RLCCr foram Pitbull, Poodle, Fila Brasileiro, Yorkshire, Rotweiler, Labrador e Lhasa Apso. Como neste estudo não houve atendimento de animais castrados antes da puberdade que apresentaram RLCCr, faz-se necessária a pesquisa da influência da castração em animais pré-púberes na ocorrência de RLCCr.

Palavras chave: castração, gênero, idade, porte, raça

\section{Rupture of the cranial cruciate ligament of dogs treated at the veterinary hospital of the Anhembi Morumbi University}

\begin{abstract}
The aim of this study is identify risk factors such as race, age, sex, body weight and spaying/ neutering in dogs that suffered rupture of the cranial cruciate ligament (RCCL), treated at Hovet Universidade Anhembi Morumbi. Records of cases of dogs treated with RCCL were surveyed in the 2009 - 2015 period. In the period studied, 382 animals were treated with RCCL, of which 189 were females and 193 males. There is no statistically significant difference between males and females at the age of neutering, age at rupture and in the interval between neutering and RLCCr. But there was a statistically significant difference in the average age at RCCL of animals over $20 \mathrm{~kg}$ compared to the others.It was concluded that there is influence of the animal weight in the occurrence of RLCCr, since animals with more than $20 \mathrm{~kg}$ presented the RLCCr with a lower age than the smaller animals. The races most affected by RLCCr were Pitbull, Poodle, Fila Brasileiro, Yorkshire, Rotweiler, Labrador and Lhasa Apso. As in this study there was no treatment of spaying/ neutering animals before puberty that presented RLCCr, it is necessary to investigate the influence of spaying/ neutering in prepubertal animals in the occurrence of RLCCr.
\end{abstract}

Keywords: spaying/ neutering, gender, age, size, race 


\title{
Ruptura del ligamento cruzado craneal en perros del hovet de la Universidad Anhembi Morumbi
}

\begin{abstract}
RESUMEN. El objetivo del presente trabajo fue identificar factores considerados de riesgo, como raza, edad, sexo, peso corporal y castración en perros que sufrieron ruptura del ligamento cruzado craneal (RLCCr), atendidos en el Hovet Universidad Anhembi Morumbi. Se investigaron las fichas de los perros con RLCCr atendidos en el período de 2009 a 2015. En este período fueron atendidos 382 animales con RLCCr, de los cuales 189 hembras y 193 machos. No hubo diferencia estadísticamente significativa entre machos y hembras con RLCCr, entre edad de la ruptura entre los sexos y entre intervalo de la castración y RLCCr, pero hubo diferencia estadísticamente significativa en la edad a la ruptura de los animales con peso superior a $20 \mathrm{~kg}$ en relación a los demás. Se concluyó que hay influencia del peso del animal con la frecuencia de la RLCCr, pues animales con más de $20 \mathrm{~kg}$ presentaron la RLCCr con una edad inferior a la de los animales de porte menor. Las razas más afectadas por la RLCCr fueron Pitbull, Poodle, Fila Brasileño, Yorkshire, Rotweiler, Labrador y Lhasa Apso. Como en este estudio no hubo atención de animales castrados antes de la pubertad que presentaron RLCCr, se hace necesaria la investigación de la influencia de la castración en animales pre púberes en casos de RLCCr.
\end{abstract}

Palabras clave: Castración, género, edad, porte, raza

\section{Introdução}

A ruptura do ligamento cruzado cranial (RLCCr) é uma das lesões mais comuns, sendo responsável por instabilidade da articulação do joelho, o que resulta em claudicação e desenvolvimento de doença articular degenerativa (DAD) (Piermattei \& Flo, 1999; Bregadioli et al., 2014; Chung et al., 2016). Este ligamento é o mais acometido, pois está relacionado ao movimento articular, impedindo o deslocamento cranial da tíbia em relação ao fêmur, limitando a rotação interna e hiperextensão do joelho. Os cães de grande porte estão mais predispostos a RLCCr do que as raças de pequeno porte, ocorrendo principalmente em cães Rottweiler e Labrador Retriever, e com idade entre 5 e 7 anos. Em cães de raças de pequeno porte esta afecção também pode ser observada, embora a idade de ocorrência seja entre 7 e 10 anos e quase sempre com uma degeneração ligamentar pré-existente (Cardoso Junior et al., 2004; Vasseur, 2007; Dal-Bo et al., 2014; Köing et al., 2016).

Em um trabalho retrospectivo envolvendo 3218 cães com diagnóstico de RLCCr, Slauterbeck et al. (2004) observaram que as fêmeas que sofreram ovariohisterectomia e machos orquiectomizados tiveram uma prevalência maior de ruptura do ligamento cruzado anterior do que os cães sexualmente intactos. Cães de grande porte tiveram um aumento da prevalência da lesão do ligamento cruzado anterior em comparação com cães pequenos ou de porte médio, fato também constatado em outros dois trabalhos (Matera et al., 2007; Bach et al., 2015). Já em relação ao sexo, Matera et al. (2007) e Bach et al. (2015) observaram que as fêmeas foram mais acometidas por RLCCr do que os machos e em relação ao estado reprodutivo, as fêmeas e animais não castrados foram mais acometidos.

O objetivo do presente trabalho foi identificar fatores considerados de risco, como raça, idade, sexo, peso corporal e castração em cães que sofreram ruptura do ligamento cruzado cranial, atendidos no Hovet Universidade Anhembi Morumbi.

\section{Material e métodos}

Foram pesquisadas as fichas dos animais atendidos com ruptura do ligamento cruzado cranial (RLCCr) no período de 2009 a 2015 no HOVET- UAM, para obtenção de dados como: idade do animal, raça, peso, idade quando começaram os sintomas, se castrado, idade à castração, e telefone do proprietário para pesquisa dos dados faltantes na ficha do animal. Foi feita a análise dos dados, correlacionando a idade do aparecimento dos sintomas em animais castrados e não castrados, assim como a avaliação da raça e o peso do animal com maior incidência da ruptura do ligamento cruzado cranial. Para a análise estatística dos dados obtidos, foi utilizado o teste de qui-quadrado.

\section{Resultados e discussão}

No período pesquisado, foram atendidos 382 animais com RLCCr, dos quais 189 fêmeas e 193 
machos, concordando com Souza et al. (2011), que também não observou diferença da ocorrência de RLCCr entre os sexos. Este número representou $0,37 \%$ de todos os atendimentos do HOVET no período (104.006 atendimentos). Dos animais atendidos com RLCCr, não foi possível obter as informações necessárias nas fichas de 44 fêmeas e 18 machos, portanto o grupo de animais analisado foi de 145 fêmeas e 175 machos. Destes, 69 machos e 88 fêmeas eram castrados.

$\mathrm{O}$ número de machos castrados com RLCCr (69) foi menor que o numero de machos intactos (106), concordando com os resultados obtidos por Matera et al. (2007) e Bach et al. (2015). Porém em relação às fêmeas ocorreu o inverso, já que o número (88) de fêmeas castradas com RLCCr foi maior que o de intactas (57) (Figura 1).

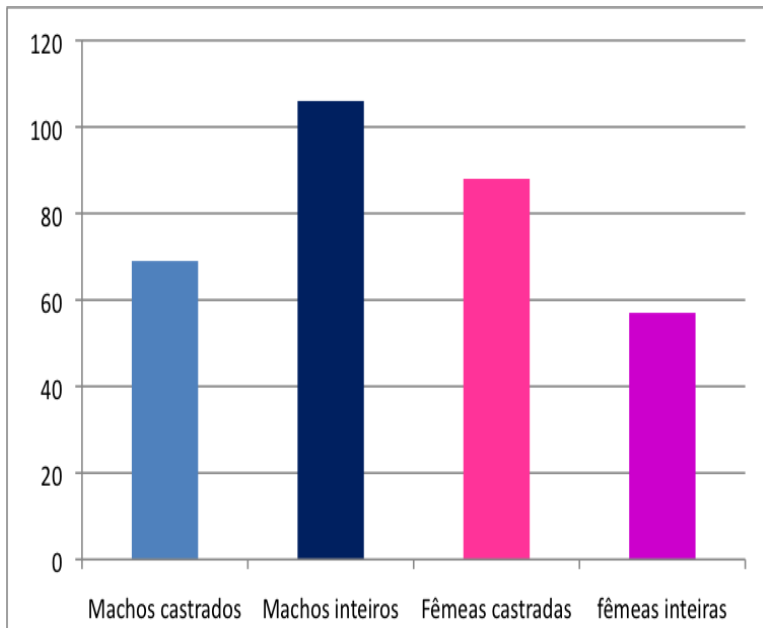

Figura 1. Número de animais inteiros e castrados com RLCCr, atendidos no HOVET-UAM

Porém Slauterbeck et al. (2004) afirmam que os animais castrados, independente do sexo, apresentam maior incidência de RLCCr que animais inteiros, tal fato concorda em parte com o presente estudo, já que apenas fêmeas castradas apresentaram maior incidência da afecção. Este fato pode ser explicado pela maior frequência da realização da castração em fêmeas do que em machos, para prevenção de tumores de mama e piometra (Duval et al., 1999; Lampman et al., 2003).

A idade à castração dos machos foi de $4,9 \pm 3,9$ anos, e a das fêmeas foi de $6,09 \pm 3,6$ anos, não havendo diferença estatisticamente significativa $(\mathrm{p}=0,235)$ entre elas (Figura 2).

É importante salientar que no período pesquisado não houve atendimento no HOVETUAM de animais com RLCCr que haviam sido castrados antes da puberdade. Não houve diferença estatisticamente significativa $(\mathrm{p}=0,186)$ da idade à ruptura do ligamento cruzado nos machos castrados $(8,19 \pm 4,06$ anos) e nas fêmeas castradas (7,33 \pm 4 anos) (Figura 3$)$.

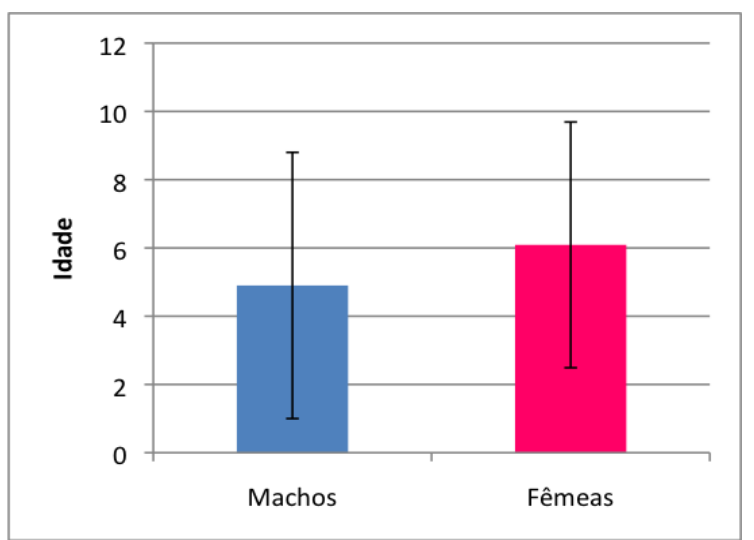

Figura 2. Idade à castração de machos e fêmeas com RLCCr, atendidos no HOVET- UAM.

O intervalo entre a castração e a ruptura do ligamento cruzado nos machos foi de 4,9 2,91 anos e não diferiu estatisticamente $(\mathrm{p}=0,84)$ do intervalo nas fêmeas $(3,92 \pm 2,57$ anos) (Figura 4) .

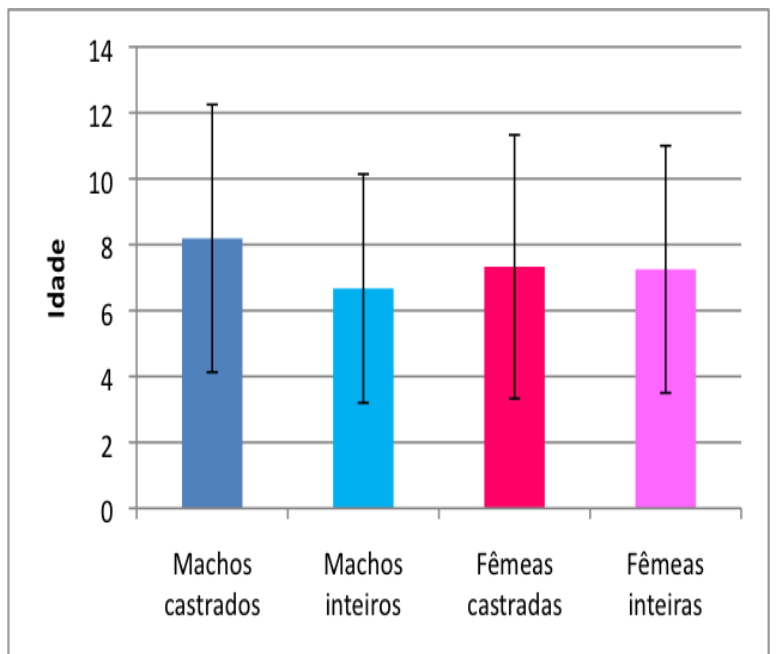

Figura 3. Idade à ruptura do ligamento cruzado cranial em machos e fêmeas atendidos no HOVET-UAM.

Em relação aos animais inteiros, a idade à RLCCr foi de 6,67 $\pm 3,47$ anos nos machos e 7,25 $\pm 3,75$ anos nas fêmeas, não há diferença estatisticamente significativa entre elas $(p=0,326)$, e também não há diferença estatisticamente significativa entre a idade à RLCCr entre animais castrados e não castrados.

Os cães das raças Pitbull (36 animais), Fila Brasileiro (30 animais), Rotweiler (24 animais) e Labrador (23 animais) foram os mais acometidos 
pela RLCCr dentre as raças de porte maior. Dentre os cães de porte menor, os mais acometidos foram os das raças Poodle (48 animais), Yorkshire (30 animais) e Lhasa Apso (24 animais) (Figura 5).

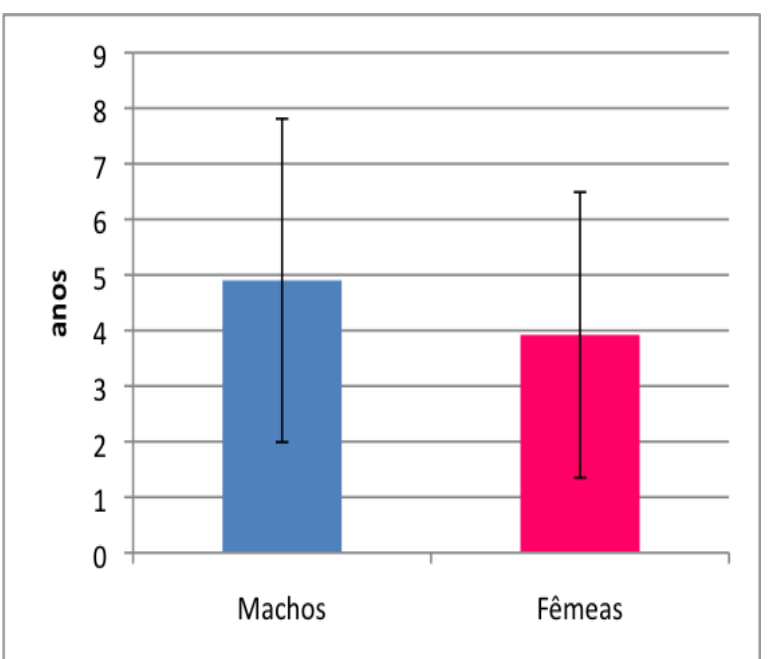

Figura 4. Intervalo (em anos) entre a castração e a RLCCr, em machos e fêmeas atendidos no HOVET-UAM.

Souza et al. (2011) descrevem a maior incidência de RLCCr em animais das raças Pitbull, Boxer, Poodle e Rotweiller e Bach et al. (2015) encontraram em seu trabalho maior número de animais das raças Pitbull e Poodle com RLCCr, resultados que estão concordando com este trabalho. $\mathrm{O}$ último trabalho ainda, ressalta que o modismo de raças em certos locais e tempo podem variar e por isso certas raças não são citadas em alguns trabalhos, como é o caso do cão da raça Pitbull, que é representativo no Brasil, mas em alguns países não. Em relação a esse fato, Duval et al. (1999) afirmam que as raças de cães com predisposição à RLCCr são Mastif Napoletano, Akita, São Bernardo , Rottweiler, Chesapeake Bay Retriever, Labrador Retriever, e American Staffordshire Terrier.

Os animais com RLCCr foram divididos em 3 categorias em relação ao peso: $23 \%$ eram animais de até $10 \mathrm{~kg}, 20 \%$ tinham de 10 a $20 \mathrm{~kg}$ e $57 \%$ tinham peso acima de $20 \mathrm{~kg}$. Houve diferença estatisticamente significativa em relação à idade média à RLCCr dos animais com mais de $20 \mathrm{~kg}$ em relação aos demais. No estudo realizado por Matera et al. (2007) e Bach et al. (2015) foi observado também, maior ocorrência de RLCCr em animais adultos de grande porte, pesando entre 26 e $35 \mathrm{~kg}$, sendo que os animais mais jovens de raças de grande porte e gigantes, foram os mais acometidos.

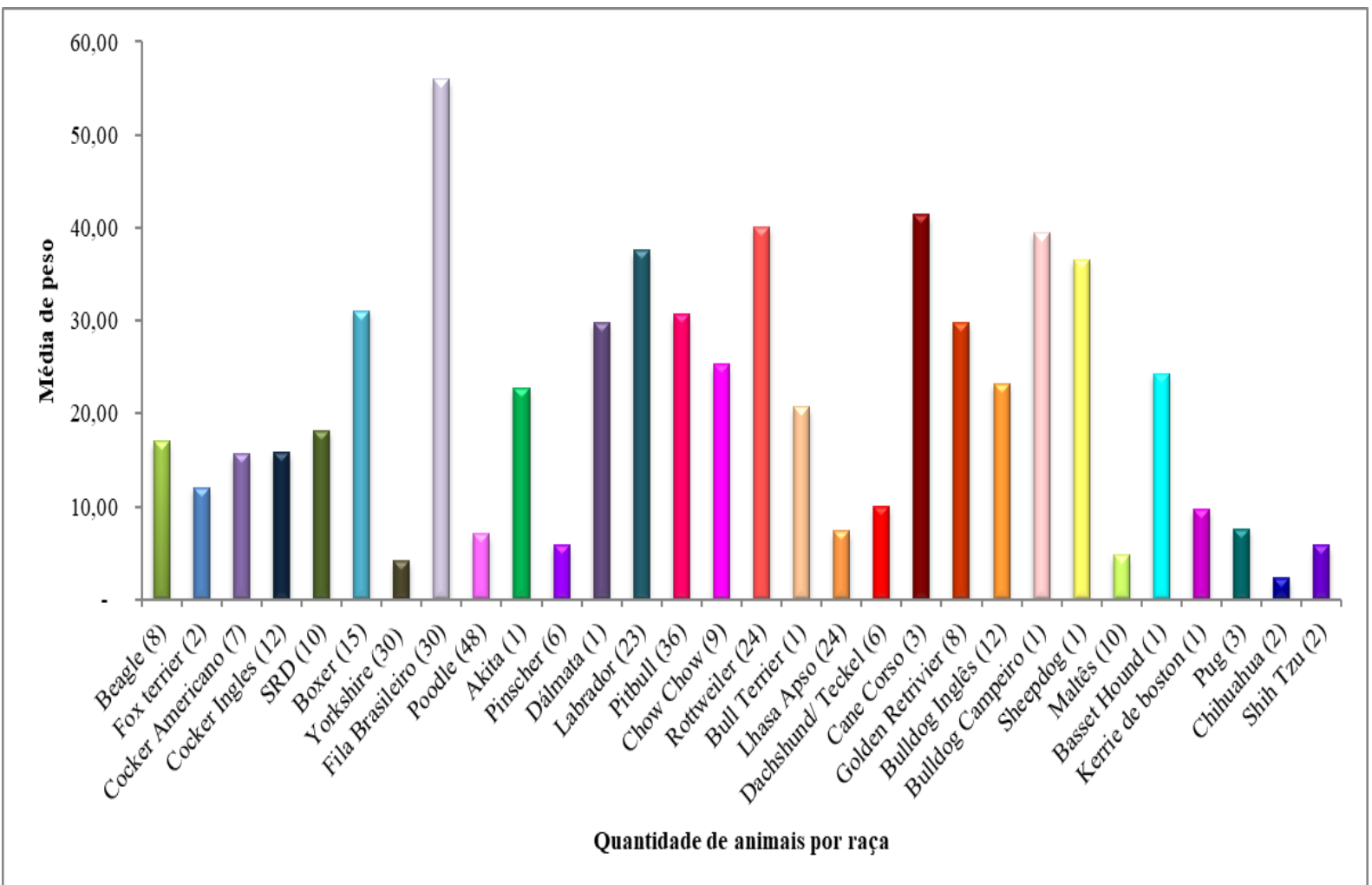

Figura 5. Número de animais por raça, acometidos pela RLCCr, atendidos no HOVET-UAM. 
Nos animais com peso acima de $20 \mathrm{~kg}$, a idade média à RLCCr foi de 5,87 $\pm 3,46$ anos, já nos animais com peso entre 10 e $20 \mathrm{~kg}$ foi de $8,23 \pm$ 4,24 anos e nos animais com peso abaixo de $10 \mathrm{~kg}$ a idade à RLCCr foi de $8,74 \pm 3,47$ anos (Figura 6), demonstrando que há maior ocorrência de RLCCr em animais mais jovens de raças de grande porte. Porém, ainda segundo Bach et al. (2015), em seu estudo, o ganho de peso dos animais não foi relacionado diretamente com a castração, mas sim com mudanças comportamentais, de hábitos alimentares e da frequência de atividades físicas. Esses fatores podem ter alguma ligação com a cirurgia, mas seriam necessários estudos mais aprofundados para esta afirmação.Vários fatores podem contribuir para a obesidade, incluindo genética, raça, idade, a quantidade de atividade física, distúrbios hormonais, medicamentos e fatores relacionados com os proprietários e mudanças no hábito e manejo alimentar, aumentando o risco de várias complicações na saúde do animal (Zerlotiini et al.,2012).

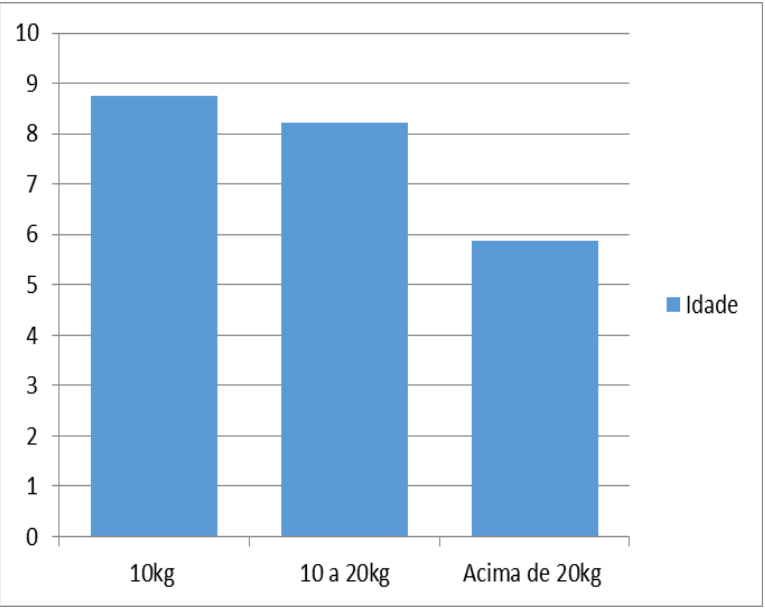

Figura 6. Relação entre peso, e idade à RLCCr, , em machos e fêmeas atendidos no HOVET-UAM.

\section{Conclusão}

De todos os parâmetros avaliados, como sexo, castração, peso e raça, concluiu-se que há influência do peso do animal na ocorrência da RLCCr, pois animais com mais de $20 \mathrm{~kg}$ apresentaram a RLCCr com uma idade inferior à dos animais de porte menor. As raças mais acometidas pela RLCCr foram Pitbull, Poodle, Fila Brasileiro, Yorkshire, Rotweiler, Labrador e Lhasa Apso. Como neste estudo não houve atendimento de animais castrados antes da puberdade que apresentaram RLCCr, faz-se necessária a pesquisa da influência da castração em animais pré-púberes na ocorrência de RLCCr.

\section{Referências Bibliográficas}

Bach, M., Junior, J. A. V., Tasqueti, U. I., Pimpão, C. T., Prado, A. M. B. \& Michellotto, P. V. 2015. Estudo retrospectivo de ruptura do ligamento cruzado cranial: 32 casos (2006 a 2012). Ciências Agrárias, 36(3), 1409-1418.

Bregadioli, T., Mota, F. C. D., Eurides, D., Faria, L. M., Dias, R. C. \& Souza, L. A. 2014. Uso da técnica Tight Rope modificada em cães com ruptura do ligamento cruzado cranial. Revista Brasileira de Ciência Veterinária, 21(2), 9095.

Cardoso Junior, R.B., Luz, L. R., Schamall, R. F., Cuiias, A. B. F., Silva, D. B., Maia, P. C. S. \& Vieira, F. A. F. 2004. Avaliação do reparo da ruptura do ligamento cruzado cranial em cães (canis familiaris): uso de uma nova técnica extracapsular com fio de nylon e grampo de aço. Brazilian Journal of veterinary Research and Animal Science, 41, 172-173.

Chung, D. G., Santos, R. M., Morato, G. O., Rocha, A. G., Padilha Filho, J. G. \& Sampaio, G. R. 2016. Transposição e avanço da tuberosidade tibial para tratamento da luxação medial de patela associada à ruptura do ligamento cruzado cranial em cão de pequeno porte: relato de caso. UNIMAR Ciências, 25(12), 08-13.

Dal-Bo, I. S., Ferrigno, C. R. A., Caquias, D. F. I, Della Nina, M. I.; Ferreira, M. P., Figueiredo, A. V., ... Ferraz, V. C. M. 2014. Correlação entre ruptura de ligamento cruzado cranial e lesão de menisco medial em cães. Ciência Rural, 44(8), 1426-1430.

Duval, J. M., Budsberg, S. C., Flo, G. L. \& Sammarco, J. L. 1999. Breed, sex and body weight as risk factors for rupture of the cranial cruciate ligament in young dogs. Journal of the American Veterinary Medical Association, 215(6), 811-814.

König, H. E., Liebh, H. G. \& Maierl, J. 2016. Membros pélvicos ou posteriores. In: König, H. E. \& Liebich, H. G. Anatomia dos animais domésticos (6a ed., p 223-288). Ed. Artmed, São Paulo, Brasil.

Lampman, T. J., Lund, E. M. \& Lipowitz, A. J. 2003. Cranial cruciate disease: Current status of diagnosis, surgery, and risk for disease. Veterinary and Comparative Orthopaedics Traumatology,16(3), 122-126.

Matera, J. M., Tatarunas, A. C., Oliveira, R. M. D., Brugnaro, M. \& Macchione, R. F. 2007. 
Estudo epidemiológico retrospectivo de cães portadores de ruptura do ligamento cruzado cranial: 323 casos (1999 a 2005). Brazilian Journal of veterinary Research and Animal Science, 44(1), 88-95.

Piermatteld, D. \& Flo, G. L. 1999. A articulação fêmuro-tibio-patelar. In: Piermattei, D. \& Flo, G. L. Manual de ortopedia e tratamento das fraturas dos pequenos animais (3a ed., p. 480538). Manole, São Paulo, Brasil.

Slauterbeck, J. R., Pankratz, K., Xu, K. T., Bozeman, S. C. \& Hardy, D. M. 2004. Canine ovariohysterectomy and orchiectomy increases the prevalence of ACL injury. Clinical Orthopaedics \& Related Research, 429, 301305.

Souza, M. M. D., Rahal, S. C., Padovani, C. R., Mamprim, M. J. \& Cavini, J. H. 2011. Afecções ortopédicas dos membros pélvicos em cães: estudo retrospectivo. Ciência Rural, 41(5), 852-857.
Vasseur, P. B. 2007. Articulação do joelho. In: Slatter, D. Manual de cirurgia de pequenos animais ( $3^{\text {a }}$ ed., p. 2090-2133). Manole, São Paulo, Brasil.

Zerlotiini, M. F., Pontes, K. C. S. \& Pinto, R. 2012. Análise da variação de peso corporal e do comportamento de cães após ováriohisterectomia e orquiectomia eletiva. Revista Científica Univiçosa, 3(1), 49-54.

Recebido: 20 Fev., 2018.

Aprovado: 18 Jun., 2018.

Publicado: 28 Jul., 2018.

Licenciamento: Este artigo é publicado na modalidade Acesso Aberto sob a licença Creative Commons Atribuição 4.0 (CC-BY 4.0), a qual permite uso irrestrito, distribuição, reprodução em qualquer meio, desde que o autor e a fonte sejam devidamente creditados. 